\title{
A study on the application of control chart in healthcare
}

\author{
Yee Kam Seoh ${ }^{*}$, Voon Hee Wong ${ }^{1}$ and Mahboobeh Zangeneh Sirdari ${ }^{1}$ \\ ${ }^{1}$ Department of Mathematical and Actuarial Sciences, Lee Kong Chian Faculty of Engineering and \\ Science, Universiti Tunku Abdul Rahman, 43000 Kajang, Selangor, Malaysia
}

\begin{abstract}
The most concerning issues in the healthcare system will always be quality control and quality improvement as they are significant to the health condition of the patient. A quality statistical tool such as statistical process control (SPC) charts will be efficient and highly effective in reducing the sources of variation within the healthcare process and in monitoring or controlling improvement of the process. The control chart is a statistical process control methodology designed to evaluate the process improvement or change in the manufacturing industry and is being implemented gradually in the healthcare sector. This will enable healthcare organizations to prevent unnecessary investment or spending in any changes that sound good but do not have any positive impact on real progress or improvement. When there is greater participation of humans in healthcare, the risks of error are also greater. Control charts help determine the source of error by differentiating the common and special cause of variation, each requiring a different response from healthcare management. This paper intends to deliver an overview of SPC theory and to explore the application of SPC charts by presenting a few examples of the implementation of control charts to common issues in the healthcare sector. After a brief overview of SPC in healthcare, the selection and construction of the two widely used control charts (Individuals and Moving Range chart, U chart) were adopted and illustrated by using the example from healthcare.
\end{abstract}

\section{Introduction}

The primary concerns in the healthcare system will always be quality control and quality improvement as they would bring a significant impact on the health condition of the patients. Also, the shifts in the global demographic such as the rise of lifestyle disease, lifestyle expectancy, and aging of the population have led to the anticipation of demand growth for quality healthcare services in the future [1]. Hence, it is critical for the authorities to maintain a secure and effective healthcare system. According to the researcher Arthur [2], about 1\% of the patients will die and $6 \%$ of the patients will be totally disabled due to the consequence of medical error. The research has also shown that about $5 \%$ to $10 \%$ of the patients contracted an infection during treatment; $15 \%$ of the diagnoses are incorrect; $50 \%$ of the patients are exposed to preventable harm while being treated in hospital. Thus, healthcare performance

*Corresponding author: seohyk@utar.edu.my 
needs to be constantly monitored and improved to minimize the adverse effect. To improve performance in the healthcare system, changes need to be made. To ensure the changes are positive and beneficial, the researchers will first need to define the key metrics, then collect a sufficient amount of data and interpret the information. This research will emphasize data interpretation and analysis through statistical process control (SPC) tools. SPC chart is a statistical technique that is useful in reducing the variability in the healthcare process and provides additional control over process improvement [3]. The SPC chart was originally used to improve the process and quality of goods in the manufacturing industries. Recently, it has been increasingly used in service sectors such as healthcare [3], [4]. This paper intends to deliver an overview of SPC theory and to explore the application of SPC charts by presenting a few examples of the implementation of control charts to common issues in the healthcare sector. After a brief overview of SPC in healthcare, the selection and construction of the two widely used control charts (Individuals and Moving Range (XmR) chart, U chart) were adopted and illustrated by using the example from healthcare.

\section{Problem statement}

One of the biggest challenges that healthcare organizations face in performance improvement work nowadays is the variation in healthcare processes and efficiencies [5]. According to the statistician Dr. W. Edwards Deming, the reduction of the statistical variation is the key to improve the quality and performance [6]. The growth of healthcare studies further -reaffirms the relationship between minimizing variability and improving performance [5].

A continuous quality improvement of the healthcare system requires the understanding and measuring of process variation [7]. Therefore, it is important to eliminate the inappropriate process variations and shift the clearly defined metrics in the direction of their expected standards. For instance, the variables which are significant in the healthcare sector include infection rate, waiting time, mortality rate, patient satisfaction level, medical error, response time for emergency services, and so forth. Hence, the monitoring and analyzing of these variables can lead to significant quality-related improvement. In this context, a quality statistical tool such as SPC charts can be useful in analyzing the process variation; monitoring healthcare performance; and determining whether quality improvement or deterioration is taking place [7]. Control charts are a valuable SPC tool established by physicist Walter Shewhart to identify if the variability in the manufacturing process is consistent and within the control state [8]. Recently, there is a growing interest in the healthcare sector to implement the control chart to monitor and improve the healthcare process performance. Nevertheless, since SPC is relatively new in the sector and not widely introduced in medical statistics courses or texts; therefore, research has to be conducted to study the application of control charts in healthcare.

\section{Literature review}

Several studies suggested the use of control charts as a valuable SPC tool in healthcare process improvement and management. Some research reported the SPC application helped teams differentiate special causes variation from common causes. For example, the investigators applying charts in surgical care to monitor the length of stay, mortality rate, and admission rate in intensive unit care and concluded the control charts enable the making of the surgical process stable by identifying and removing the special causes of variations [9]. Some articles reported how control charts helped teams to decide on the correct improvement 
strategy. For instance, investigators presented various examples of control charts, $U$ chart, $\bar{X}$ and S chart, $g$ chart, $p$ chart, and XmR chart, to monitor flash sterilization rate, surgical site infections, laboratory turnaround time, appointment access satisfaction, and infectious waste. The investigators ended up with the conclusion that control charts can help healthcare organizations to prevent unnecessary investment in changes that sound great but do not have any beneficial impact on improvement [10]. Since the 1990s, the gradual increase in the quantity and quality of the research regarding the implementation of SPC tools in the healthcare sector has justified that the control chart has taken a beneficial role in improving healthcare process performance such as waiting time, percentage of hospital errors, infection rate, mortality rate or death rate [11], [12], [13], [14], [15], [16]. This section first delivers the SPC theory, then followed by the control chart concept. Then, it will discuss the control chart interpretation and test rule. Lastly, it will discuss the data type and method for selecting the proper control chart.

\subsection{Statistical process control (SPC) theory}

Dr. Walter Andrew Shewhart, a well-known physicist, developed the basics of statistical process control (SPC) and the associated control chart tool to improve the industrial manufacturing process [11]. The control charts have been commonly used in the manufacturing industry over several decades since Shewhart first presented it in 1924. Within the context of the continuous quality improvement process, the control charts are increasingly extended to the service sector, including healthcare, to assess process consistency and capability; to ease the understanding of the process and to identify changes that could lead to improvements or deteriorations of quality [7], [13].

\subsection{Concept of control chart}

SPC is a continual improvement methodology that could be applied to different sorts of statistical tools to monitor and improve the production process. Its key statistical tool is known as the statistical control chart, as the application of SPC commonly requires the construction of control charts [17], [18]. According to the researchers, the charts illustrate how the production or process differs over time. Thus, we could recognize whether a process is in or out of control and whether the source of variation is caused by common causes or by special causes [13], [17].

The process variation comes from two distinct sources, common and special causes [17]. Common cause variation is unavoidable. It is a variation that occurs and exists as nature in a stable or in the control process. It can only be disclosed through consistent process learning and be reduced through system redesign [13]. Special cause variation is unnatural variation due to changes and events that have not previously been presented in the process as a standard basic [10]. It needs to be analyzed to determine the source and take appropriate measures to eliminate it. If the process does not contain any special cause variation and exhibits only common cause variation; the process is said to be stable or in control [13].

Two general approaches can be applied to improve the process through variation observation. If the process exhibits only common cause variation, a similar result will continually be generated within the statistical limits, unless the system is redesigned or restructured. On the other hand, a process that exhibits special cause variation is unstable and unpredictable. It can be improved by first eliminating the special causes to bring the process back into the controlled state. Therefore, if the aim is only to maintain the existing 
performance level, special cause variation must be avoided and eliminated [10]. Nevertheless, if the purpose is to improve the performance level, it is both important to remove the special cause variation and minimize the remaining common causes variation. Then, changes can be introduced to reshape the process structure in the desired direction.

According to Montgomery [19] the application of statistical process control charts commonly defined as involving two stages or phases (Phase I and Phase II implementation), with two distinct purposes. In Phase I, the initial phase, retrospective analysis on historical data is performed to build trial control limits that act as a baseline and could be used to verify whether the process is in-control and to evaluate if the control limits can be continually applied to monitor the future performance. Phase I control charts primarily facilitate to bring the process to a stable state, which is the critical stage of process control. If the process parameters are unidentified, a phase I implementation must be conducted before process monitoring can begin in Phase II. The Phase II control charts are used to monitor the performance and presume that a reference sample can be obtained from the corresponding Phase I implementation in which the baseline or trial control limits are evaluated.

Benneyan, Lloyd, and Plsek [10] stated control chart can identify the special cause variation more rapidly than other SPC tools. Hence, it is one of the most useful methods for determining the efficiency of a process and securing the sustainability of the continuous process improvement over time.

\subsection{Interpretation of control chart}

A control chart contains two parts: (1) a plot of time-series data, and (2) three additional horizontal lines - the centerline (average or mean), the lower control limit (LCL), and the upper control limit (UCL). To detect the significant or meaningful changes in process performance, the lower and upper control limits are set as \pm 3 standard deviations (SDs) from the centerline. If the data point falls randomly between the lower and upper control limits, it is presumed that the process exhibits only common causes variation and could be considered in the state of control. Typically, it is presumed that almost all data points will fall within \pm 3 standard deviations from the centerline if the underlying distribution is consistent. Shewhart [20] described if there is any point that goes below the lower control limit or above the upper control limit, the process is considered to be out of control. Furthermore, supplementary run rules (Section 3.4) have been developed to define the out-of-control process further [13].

\subsection{Test rule for control chart}

A general set of tests rule was suggested for special cause variation when a process is out of control [3]. They are:

1. If any points below an LCL or above a UCL.

2. If two out of three successive points on the same side of the centerline fall beyond the two sigma control limits.

3. If four out of five successive points on the same side of the centerline fall beyond the one sigma control limits.

4. A run of eight or more successive points in a row falls on either side of the centerline.

5. A trend of six or more successive points steadily decreasing or increasing.

6. A non-random or unusual pattern in the data. 


\subsection{Types of data}

Data is the fundamental of control chart. It can be classified into two types, variable data and attribute data [3]. Variable data involve continuous scale measurement, for example, body temperature, height, weight, and waiting time for surgery or consultation. Attribute data involve classification or counts, for example, mortality rate, infection rate, number of patients waiting for surgery, and status of compliance or failure to comply with medical standards. It can be either count of defectives or the count of the number of defects [13]. Defect data refers to an aspect of a product or a service unit that fails to satisfy customer requirements. The number of defects on the products or services would be counted and documented. The event of defects counting can happen to the same item several times, as there can be none, one, or a few defects in each production unit. For example, the number of dietary tray errors, the number of incorrect ID bands, and the number of patients falling. On the other hand, defective data refers to the acceptance or nonacceptance of the entire unit of production. The incident can only occur to the same item once and the result can only be binary with two values such as Yes or No. For example, categorizing an item by a specific standard such as status of compliance or non-compliance with medical standards or status of dead or alive. Table 1 shows the comparison of defect and defectives data.

Table 1. Comparison of defects and defectives data.

\begin{tabular}{|l|c|c|}
\hline \multicolumn{1}{|c|}{ Machine } & Defects & Defectives \\
\hline Machine 1 & 0 & Acceptable \\
\hline Machine 2 & 3 & Not Acceptable \\
\hline Machine 3 & 2 & Not Acceptable \\
\hline Machine 4 & 2 & Not Acceptable \\
\hline Machine 5 & 1 & Not Acceptable \\
\hline Machine 6 & 0 & Acceptable \\
\hline Total & $\mathbf{8}$ & $\mathbf{4}$ Not Acceptable \\
\hline Rate/Fraction & $\mathbf{8 / 6}$ & $\mathbf{4 / 6}$ \\
\hline
\end{tabular}

\subsection{Selection of the right control chart}

According to the researcher, seven charts are most widely applied among the various types of control charts to monitor the process performance in the healthcare sector [21]. The proper chart to apply mostly depends on the type of data that is being plotted and examined [3], [13], [17]. For variable data, three control charts can be used: XmR charts, $X$ and $R$ chart, and X and $S$ charts. For attribute data, four control charts can be used: $C$ chart, $U$ chart, $P$ chart, and NP chart [21]. These control charts are strongly recommended for Phase I implementation [17]. 


\subsubsection{Control charts for variable data}

Benneyan [22], [23] proposed $\bar{X}$ chart and S chart should apply together when the variable data is normally distributed. Moreover, other researchers proposed selecting the proper control chart by analyzing the sizes of the subgroups [21]. If the subgroup size is equivalent to one for each data point, i.e., the waiting time of a patient for surgery, the application of the $\mathrm{XmR}$ chart (X chart and Moving Range chart) commonly is suggested. If the size of the subgroup is small for each data point (more than 1 and less than or equivalent to 10) and equal in size, i.e., the waiting time of 4 patients for surgery, the application of $\bar{X}$ and $\mathrm{R}$ chart commonly is suggested. If the size of the subgroup is large for each data point (more than 10) or not equal in sizes, i.e., waiting time of 12 patients for surgery, the application of $\bar{X}$ and $\mathrm{S}$ chart commonly is suggested.

\subsubsection{Control charts for attribute data}

Benneyan [22], [23] proposed either a $U$ chart or $C$ chart should be applied when the attribute data is from Poisson distribution. When the attributed data generated from Binomial distributions, it is advised to apply either NP chart or P chart. Furthermore, other researchers suggested that the selection of the control chart for attribute data chart would depend on whether the data points are counts of defectives or counts of the number of defects [21]. If the data obtained from counts of defects, it is advised to apply either a $\mathrm{U}$ chart or $\mathrm{C}$ chart. If the size of the subgroup for every data point is fixed, i.e., every data point reflects the number of falls in a sample of 10 patients, then generally a $\mathrm{C}$ chart is proposed. If the data depends on defect per unit or rate of defects, i.e., the incidents per monthly discharge or rate of patient fall per 30 patient days, then a $U$ chart is more suitable. The $U$ chart subgroup sizes can be either fixed or variable.

If the data obtained from counts of defectives, it is advised to apply either NP chart or P chart. If the size of the subgroup for every data point is fixed, i.e., the number of medical devices does not comply with a medical standard per 50 devices monthly; then generally an NP chart is suggested. If the data depends on percent defectives or rate of defectives, i.e., the mortality rate per month, since the number of deaths can vary from month to month, then commonly a $\mathrm{P}$ chart is more suitable. The $\mathrm{P}$ chart subgroup size can be either fixed or variable. Fig. 1 displays the relationship between data types and the respective control charts. 


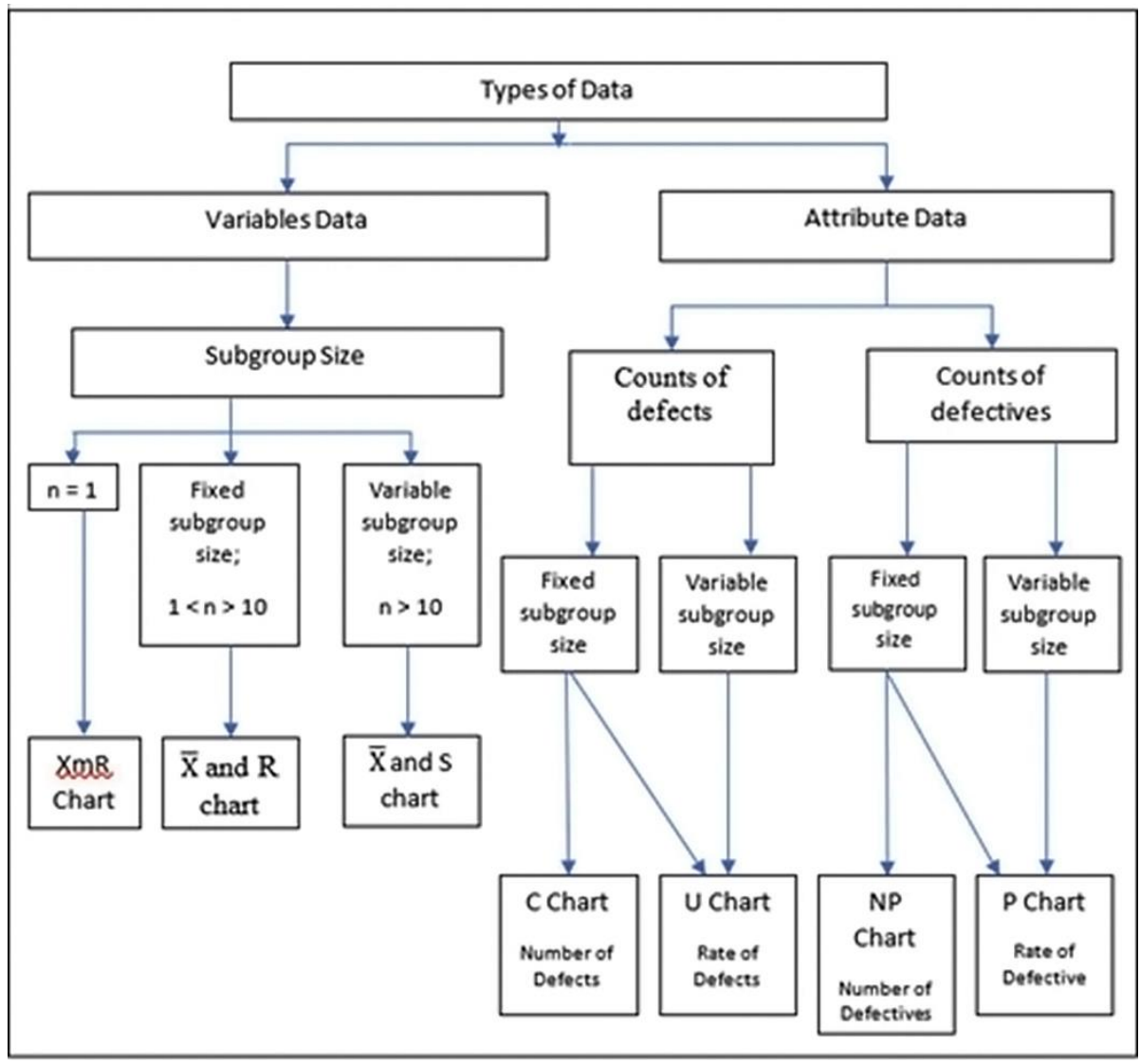

Fig. 1. The types of data and control chart [3], [13].

\section{Methodology}

This research used a quantitative method of secondary data analysis on the current healthcare sector data sets. A set of observations (or data points), also recognized as subgroups, is collected from the process from time to time, and parameter such as the ambulance response time and rate of medical devices adverse events per incident are measured from each subgroup and then plotted in chronological order with three horizontal lines (the centerline (CL), lower control limit (LCL) and upper control limit (UCL)) on a suitable control chart. The selecting of a suitable control chart relies on the type of data that is being plotted. The centerline and control limits are used to identify the significant shift in the present healthcare performance if there is any special cause variation in the process. The centerline is set at the arithmetic average of plotted data, and two control limits are set at minus and plus three standard deviations from the mean. This section will involve the formula and construction methods of the two control charts (XmR chart and U chart) that have been used frequently in healthcare.

Also, to identify the impact of a change in process, whether the changes have a beneficial impact, we can freeze the centerline and the two control limits from the historical data (Phase I implementation) and proceed to allocate the new data point on the control chart (Phase II 
implementation). A change in a quality improvement process is an attempt to introduce a special cause of variation that is done consciously and intentionally [12]. Therefore, indicators of special cause variation show that the current process varies greatly from the historical process. Then, we can use the new data to recalculate and revise the control limit. The consistency in the performance of different charts such as the XmR chart and U Chart will be explored further in the next section to recognize the process pattern with the goal to improve the quality of performance in the healthcare sector.

\subsection{The Individuals and Moving Range (XmR) charts}

The Individuals and Moving Range $(\mathrm{XmR})$ charts will be useful when the size of the subgroup used for process control is equal to one, which is, each subgroup consists of a single unit. The XmR chart has two sections: the moving range chart (MR chart) and the individual chart (X chart). $\mathrm{X}$ chart is a control chart of the $k$ data points values, $x_{1}, x_{2}, \ldots, x_{k}$, it illustrates the individual performance and the MR-chart is a control chart of the data's moving range [17], it shows the variability between the values of consecutive data points. Thus, in the MR chart, there would be one lesser data point than the $\mathrm{X}$ chart.

The first step to produce MR chart is to calculate the moving range, the differences between the consecutive data point, as $M R_{j}=\left|x_{j}-x_{j-1}\right|$, where $x_{j}$ is the value for $j^{t h}$ data points and $x_{j-1}$ is the value for $(j-1)^{\text {th }}$ data points. Then, calculate the centerline (CL), the lower control limit (LCL), and the upper control limit (UCL) by using the formula:

$$
\begin{aligned}
& \mathrm{CL}=\overline{M R}=\frac{\sum_{j=2}^{k} M R_{j}}{k-1} \\
& \mathrm{LCL}=D_{3} * \overline{M R}=0 * \overline{M R} \\
& \mathrm{UCL}=D_{4} * \overline{M R}=3.627 * \overline{M R}
\end{aligned}
$$

Table 2 shows the value of 0 and 3.627 are taken from the sample size-specific control chart constant $D_{3}, D_{4}$ for $n=2$, as given in most statistical tables and textbooks on SPC. The last step is to plot the value of $M R_{j}, \mathrm{CL}, \mathrm{LCL}$, and UCL on the same graph.

In the first step, to construct a 3 sigma $X$ chart, calculate the centerline (CL), the lower control limit (LCL), and the upper control limit (UCL) by using the formula:

$$
\begin{aligned}
& \mathrm{CL}=\bar{X}=\frac{\sum_{j=1}^{k} x_{j}}{k} \\
& \mathrm{LCL}=\bar{X}-3 \frac{\overline{M R}}{d_{2}}=\bar{X}-3 \frac{\overline{M R}}{1.128}=\bar{X}-2.66 * \overline{M R} \\
& \mathrm{UCL}=\bar{X}+3 \frac{\frac{\overline{M R}}{d_{2}}}{\overline{d_{2}}}=\bar{X}+3 \frac{\overline{M R}}{1.128}=\bar{X}+2.66 * \overline{M R}
\end{aligned}
$$

Table 2 shows the value of 2.66 is obtained by dividing 3 by the sample size-specific control constants $d_{2}=1.128$ for $n=2$, as given in most statistical tables and textbooks on SPC. The last step is to plot the value of $x_{j}, \mathrm{CL}, \mathrm{LCL}$, and UCL on the same graph and placed the graph on top of the MR chart. 
Table 2. Table of Control Chart Constants [24].

\begin{tabular}{|c|c|c|c|}
\hline \multirow{2}{*}{$\begin{array}{c}\text { Subgroup } \\
\text { Size }\end{array}$} & \multirow{2}{*}{$\boldsymbol{d}_{\mathbf{2}}$} & \multicolumn{2}{|c|}{ Factors for Control Limit } \\
\cline { 3 - 4 } & & $\boldsymbol{D}_{\mathbf{3}}(\boldsymbol{L C L})$ & $\boldsymbol{D}_{\mathbf{4}}(\boldsymbol{U} \boldsymbol{C} \boldsymbol{L})$ \\
\hline 2 & 1.128 & 0 & 3.267 \\
\hline 3 & 1.693 & 0 & 2.574 \\
\hline 4 & 2.059 & 0 & 2.282 \\
\hline 5 & 2.326 & 0 & 2.114 \\
\hline 6 & 2.534 & 0 & 2.004 \\
\hline 7 & 2.704 & 0.136 & 1.864 \\
\hline 8 & 2.847 & 0.184 & 1.816 \\
\hline 9 & 2.970 & 0.223 & 1.777 \\
\hline 10 & 3.078 & \multicolumn{2}{c}{} \\
\hline
\end{tabular}

\subsection{The U charts}

U Chart will be useful if the data is based on the rate of defects per unit or the mean number of defects per inspection unit (data represent a rate, not a count) and the size of the subgroup is inconsistent for every data point. It takes the size of the subgroup for every data point into consideration and adopts this size to generate the control limits. Since the size of the subgroup varies for each data point, then the control limit lines will be different for each data point and do not appear as a straight line.

Assume there consist of $k$ subgroups, every subgroup has $m_{j}$ inspections units. Let $U_{j}$ be the rate of defects per unit or the mean number of defects per inspections unit in $j^{\text {th }}$ subgroup and let $c_{j}=$ number of defects in $j^{t h}$ subgroup of $m_{j}$ inspections unit. Then, $U_{j}=\frac{c_{j}}{m_{j}}$.

The first step to produce a $\mathrm{U}$ chart is to calculate $U_{j}$, the rate of defects per unit, for each subgroup. Then, calculate the centerline (CL), the lower control limit (LCL), and the upper control limit (UCL) by using the formula:

$$
\begin{aligned}
& \mathrm{CL}=\bar{U}=\frac{\sum_{j=1}^{k} c_{j}}{\sum_{j=1}^{k} m_{j}} \\
& \mathrm{LCL}=\bar{U}-3 \sqrt{\frac{\bar{U}}{m_{j}}} \\
& \mathrm{UCL}=\bar{U}+3 \sqrt{\frac{\bar{U}}{m_{j}}}
\end{aligned}
$$

Set LCL at zero, if LCL < 0 . Since the size of the subgroup for each data point are different, then $\sqrt{\frac{\bar{U}}{m_{j}}}$ will vary. Hence, the control limit lines will be specific for each data point. The last step is to plot the value of $U_{j}, \mathrm{CL}, \mathrm{LCL}$, and UCL values on the same graph. 


\subsection{Research methodology}

This study will practice secondary data analysis of qualitative data in the healthcare industry. This section will illustrate how healthcare can apply the methodology of the control chart to assess the process pattern and to identify the impact of shifts in the process. Firstly, we will define the key metrics of the secondary data. Secondly, we collect the relevant National Health Service (NHS) England data. Thirdly, to analyze and interpret the data, we require to select a suitable control chart according to the data type. To examine the data and to recognize the effect of a process change, we need to define the baseline parameters (or trial control limits) based on the past-period data (data before the period of a change). Before we freeze the centerline and control limits from the past-period data (Phase I implementation), we need to make sure the baseline process is in-control or stable, if any out-of-control signal is indicated, we need to bring the baseline process back into the control state. If the process is in-control, then the baseline limits can be frozen and the new data continue to be plotted on the control chart to assess if a process change has enhanced the stable process (Phase II implementation). If an out-of-control signal is detected, it indicates that a new process is substantially different from the past-periods process. Then, using the new data (data after the time of a change), we can recalculate the new control limits.

\section{Examples - results and discussions}

The control chart, which was created for the manufacturing process, is now being increasingly implemented in the healthcare sector to monitor the performance of the healthcare process. The following examples show the underlying principles, the scope of implementation, and versatility of control charts as a tool for monitoring or controlling the statistical process and data analysis.

\subsection{Ambulance response time}

\subsubsection{Introduction}

In 2016, a government-funded medical services and healthcare in England, the National Health Service (NHS) reported that the ambulance services are struggling to reach patients with life-threatening conditions quickly enough as the healthcare service system is facing difficulties to deal with the growing demand for emergency care or service. The emergency 999 calls will be handled by the call handlers to determine or assess the urgency of the calls and appropriately assign ambulance resources [25], [26]. Historically, the target of the ambulance response needs call handlers to determine and assess the condition and location of the patients within 1 minute, from receiving the call to sending an ambulance vehicle or crew to the scene [26]. The ambulance should reach in a mean time of 8 minutes if the call is an emergency or immediately life-threatening [27]. However, the NHS England discovered the 1-minute response target is causing major inefficiencies to the ambulance service system as the if call handlers were not able to properly assess the emergency condition of patient within 1 minute of receiving a call, but due to accident \& emergency departments (A\&E) have to hit response time targets within 8 minutes to reach patient's location, the crew had no choice but to be dispatched within the time constraints [28]. Multiple vehicles will often be dispatched to meet the 8-minutes response target, implying that some crews need to stand down before they reach patients. Moreover, time and resources will be wasted when it is 
assessed that the patient is not in urgent need of immediate medical care as the ambulance will be recalled. Thus, NHS England launched a new set of ambulance guidelines across the country in July 2017 to ensure the priority is given to the most critical cases, the sickest patients obtain the fastest ambulance response and the most proper response is given to each patient for the first time [27]. The restructured system is a triage system, in which calls are triaged into four categories according to the condition of the patient and the need for urgent and immediate medical response [29]. For life-threatening injuries or illnesses, such as cardiac arrest or respiratory, which require the urgent and immediate response are classified as Category 1, paramedics and ambulance are now expected to reach the patient in meantime of 7 minutes. Category 2 is classified for emergency calls or any potentially severe conditions, such as stroke and chest pain. Category 3 is classified for urgent but not immediately life-threatening (i.e., pain control or uncomplicated diabetics) calls. Lastly, Category 4 is classified for non-urgent (i.e., stable clinical cases) calls. A new set of pretriage questions are given to the call handlers with more time to assign the incidents to one of the response categories and to classify those patients who need the quickest and most accurate medical attention [30]. Instead of sending multiple vehicles to one call, a new triage system is now sending one vehicle and providing the appropriate and right response to the patient. This will help free up paramedics and ambulances to respond to other calls. A decades-old system is updated by the new system and the new standard or guidelines was slowly rolled out across the country, and all England ambulance trusts completed the transition to the new response performance guidelines or standards and were able to report on new systems metrics by April 2018 [31]. A control chart will be used to monitor the response time of the ambulance and to assess how the process has changed with the introduction of the new system.

\subsubsection{Methods}

On 20 June 2018, the existing ambulance response time data for Category 1 of NHS England from August 2017 to May 2019 were extracted using Microsoft Excel 2016 from the NHS database. Since August 2017, NHS England began to collect and summarize the data from all of England's eleven ambulance trusts, and then released the data on the latest ambulance system metrics monthly. The total response hours (from receiving the call to the vehicle reach the patient's position) and the number of incidents is measured monthly, mean response time is calculated by dividing the number of incidents by total response hours and all data will be analyzed using Minitab 18. XmR Chart (based normal distribution) was chosen for monitoring of ambulance mean response time and to evaluate whether new system implementation has enhanced the process.

\subsubsection{Data discussion}

Before introducing the new ambulance system, the baseline period, from August 2017 to March 2018, the average ambulance response time per month was approximately 8.408 minutes and the process showed to be in a state of control. Fig. 2 illustrates the XmR control chart with the $\mathrm{CL}$ at $\bar{X}=8.408, \mathrm{LCL}=7.210$, and $\mathrm{UCL}=9.606$. The monthly average response time for the ambulance is plotted on the $\mathrm{X}$ chart (chart above) and the monthly moving range is plotted on the Moving Range chart (chart below). There is no indication that the process for this baseline period is out of control, thus these limits can be implemented to check whether the change in a process has improved the stable (in statistical control) process (Phase 
II study). Hence, the continuation of data after new system implementation, from April 2018 to May 2019 (Sample 9 to 22) is plotted in Fig. 2.

Any changes or measures in an improvement scheme are intentional attempts to introduce a special cause variation. From the examination of Fig. 2, a special cause variation due to Rule 3 is detected, which probably a sign that the process might have measurably shifted. It indicates that the process after introducing the new system showed a decline (special cause variation) to an average response time of about 7.2 minutes for ambulances. Since the process has shifted, the control limits will be revised based on the data after the new system implementation. The revised control limits as shown in Fig. 3 for $\mathrm{X}$ chart are $\mathrm{CL}=\bar{X}=7.250$, $\mathrm{LCL}=6.962$ and $\mathrm{UCL}=7.538$ and for MR chart are $\mathrm{CL}=\overline{M R}=0.108, \mathrm{LCL}=0$ and $\mathrm{UCL}$ $=0.354$. However, from the examination of Fig. 3 , there are 4 points above the UCL and 2 points below the LCL in the X chart. Analysis of the data for the first 4 months (April 2018 - July 2018) shows that ambulance trusts are still in the middle of assessing and adapting changes following the new system introduction during this period. Hence, removing these 4month data and recalculating the centerline and control limits seems logical. Fig. 4 shows the $\mathrm{XmR}$ chart with these new revised parameters. From the analysis of Fig. 4, all data points fall within the new control limit; therefore, at this new stage, the process is stable and in a state of control. The new system aims all ambulance trusts to respond with an average time of 7 minutes for Category 1 call; the control chart displays a significant enhancement in the reduction of the average response time, from $\bar{X}=8.408$ to $\bar{X}=7.108$, soon after the implementation of the new system. Despite the improvement in response time after the changes in the system process, the average $\bar{X}=7.108$ is still high as compared to the target of average 7-minutes response time. However, Fig. 4 indicates the standard of the average response time of 7 minutes was first reached during the period from March 2019 to May 2019 (sample 20 to 22). Hence, control charts can be applied consistently to monitor the sustainability of continuous improvements in the healthcare process by identifying and eliminating any future special cause variation that could induce an increase in average ambulance response time. 


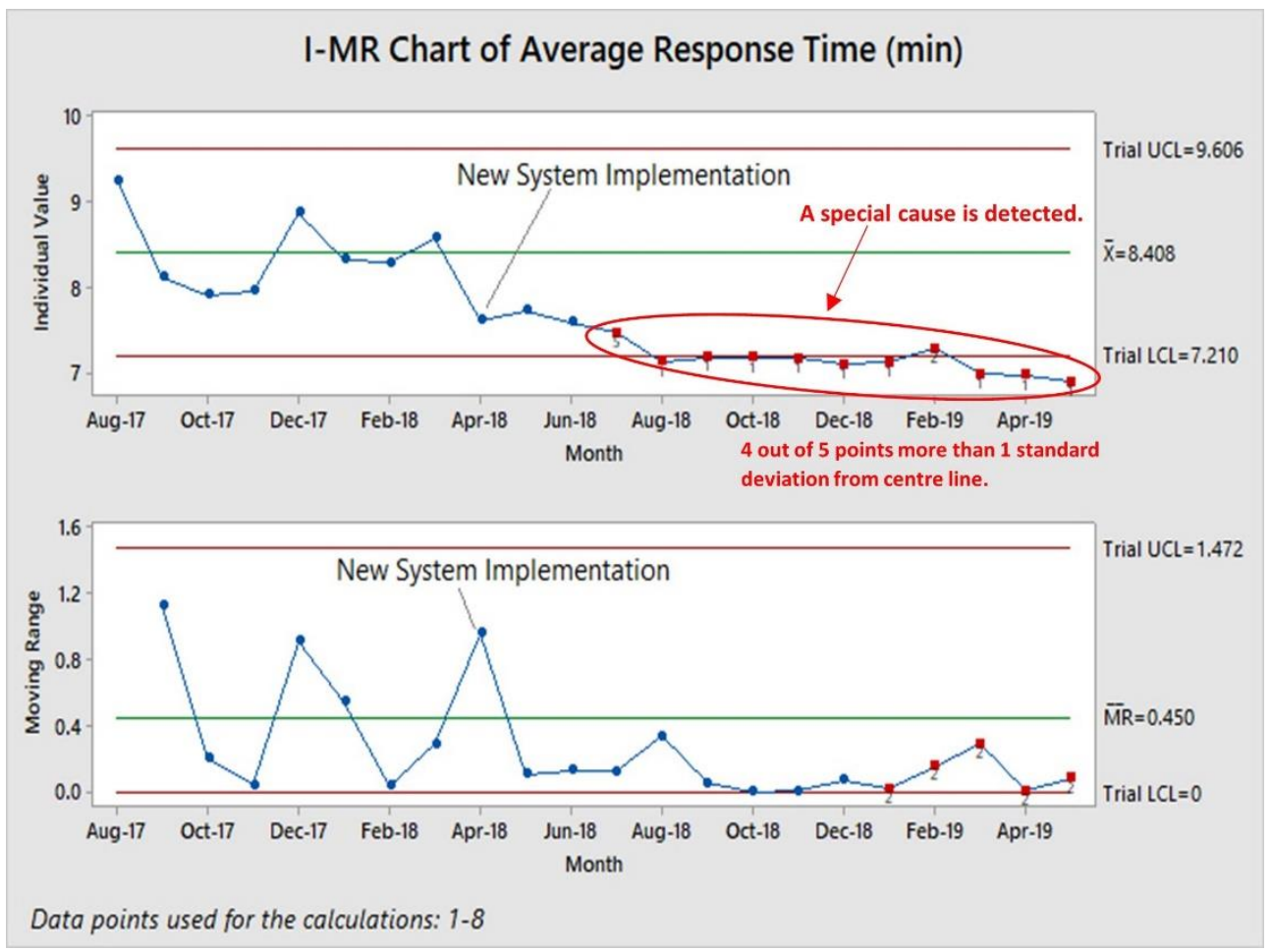

Fig. 2. XmR Control Chart for Ambulance Response Time: baseline compared with the period following implementation of the new ambulance system.

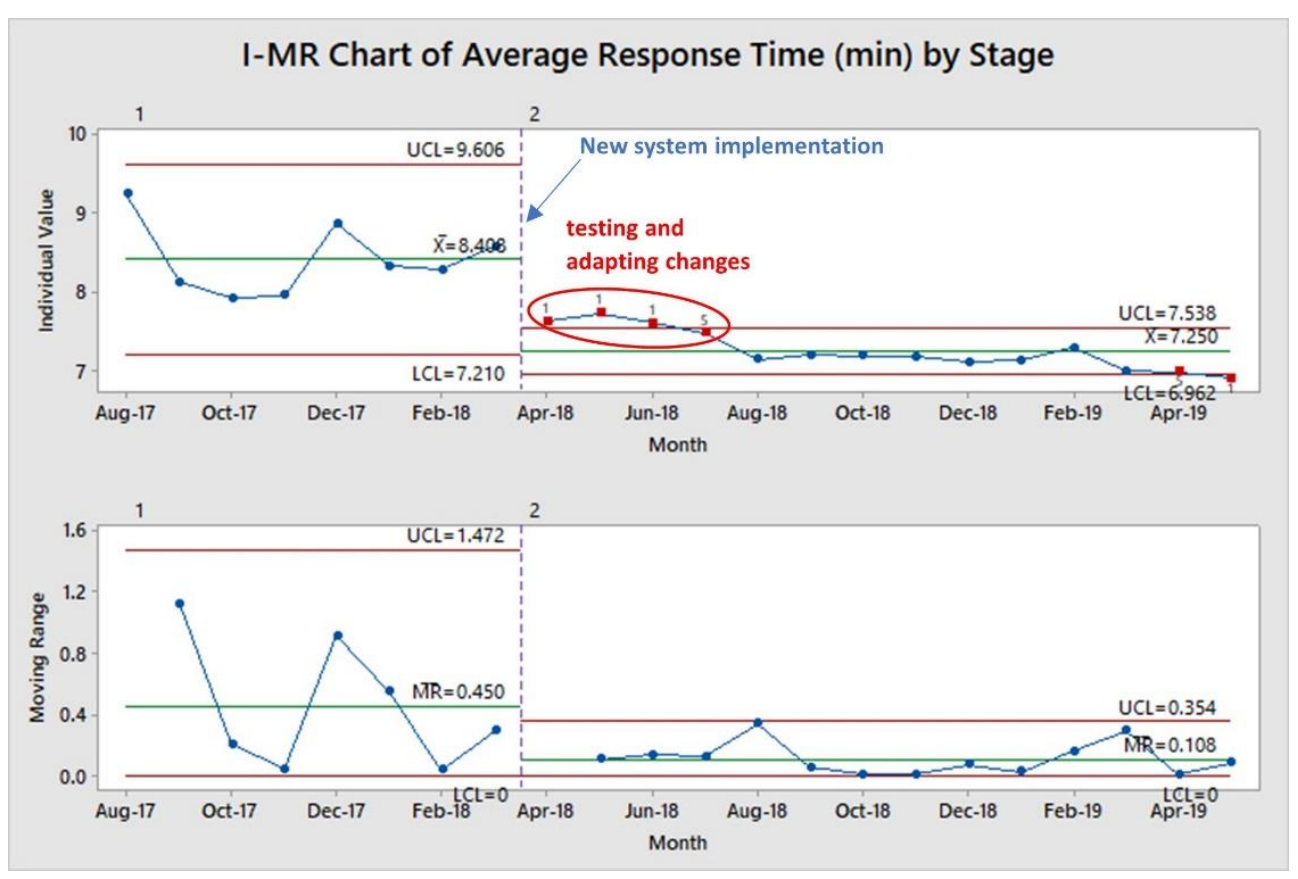

Fig. 3. Revised control limits on the average ambulance response time XmR control chart. 


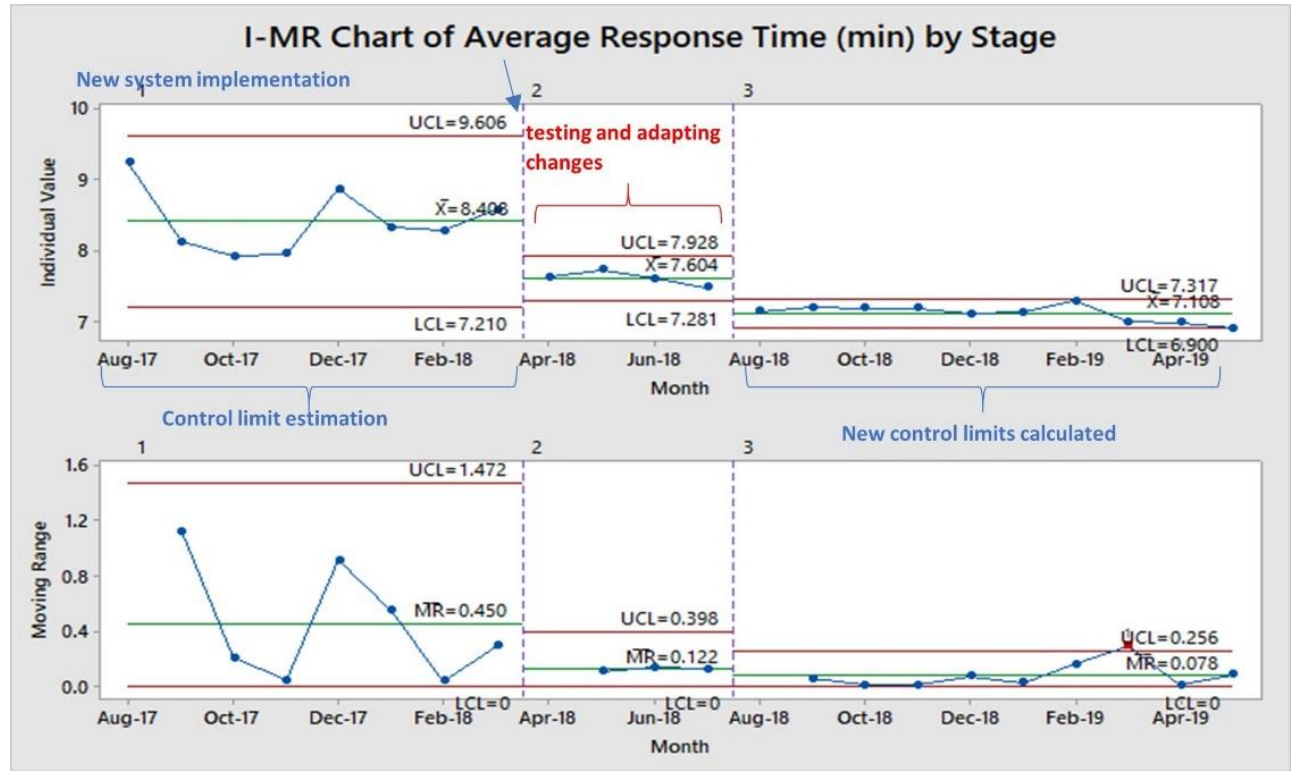

Fig. 4. New control limits on the average ambulance response time XmR control chart.

\subsection{Medical devices/equipment adverse events}

\subsubsection{Introduction}

Medical equipment and devices are the trading tools for healthcare professionals, and they play a vital role in the treatment, diagnosis, and rehabilitation in surgery, in hospitals, and at home [32], [33]. Medical devices or equipment range from low-risk, simple devices such as thermometers and disposable gloves to high-risk, sophisticated devices such as implantable pacemakers, breast implants, and defibrillators that are used to support and maintain life. Medical devices are categorized into three classes, which are Class I (lowest or minimal risk devices such as tongue depressors, elastic bandages, and dental floss), Class II (devices which present medium risks such as ultrasound devices, endoscopes, and wheelchairs), and Class III (devices which present high risk and used to sustain life such as defibrillators and implantable pacemakers). Medical equipment and devices being used in growing numbers throughout the year and demand for innovative and complex products continue to increase on the market globally [34]. There is always a danger that unavailability or failure of such equipment and devices; or failure of related procedure may result in injury, life threats, or death. One of the adverse events causing global concern for medical devices was the incident with the defective breast implant scandal produced by Poly Implant Protheses (PIP), a French company [35]. According to NHS Choices [36], the implants were found to contain unregulated silicone which was intended for industrial use, and the news on the investigation into this incident was extensively published in the media in December 2011. Around 300,000 women around the world have been affected by the scandal including France, South Africa, England, and so forth.

The UK government has conducted a rapid investigation into the safety and quality of medical devices following the tragic case of defective breast implants. Hence, the Department 
of Health launched a report in May 2012 to enhance the patient safety, which contain the recommendation below [37]:

(i) Maximize reports of adverse events involving medical devices and make certain that reports are of good quality for learning from the occurring events;

(ii) All parties (healthcare professionals, practitioners, industry, and patients) must engage in the alert system as equal partners with the sole intention of minimizing adverse events on the medical devices;

(iii) The Medicine and Healthcare products Regulatory Agency (MHRA) needs to regularly review its activities to ensure that all it does is aligned with this intention and to promote this common interest among all those stakeholders involved in the alert system.

In April 2014, MHRA has built a partnership with NHS England to launch a new framework to assist hospitals to enhance medical devices incidents reporting and data collection, improve safety learning in medical devices and guide practice to mitigate harm from medical device incidents [37], implementation of the framework need to be done by $19^{\text {th }}$ September 2014. This strategic partnership includes some initiatives as follow:

(i) Established a new integrated National Learning and Reporting System (NRLS) to exchange data on adverse events between NHS and MHRA on medical devices.

(ii) Set up a National Medical Devices Safety Improvement Network as a new platform to review the identified and possible safety concerns, examine trends and patterns of adverse events; and identify metrics to enhance the safety of medical devices.

(iii) Improve the NRLS and MHRA feedback system to enhance learning at the national level.

The Medicine and Healthcare products Regulatory Agency (MHRA) is a UK government agency responsible for ensuring the proper functioning and adequate safety of the medicines and medical devices or equipment [38]. Health experts and experts in medical devices will evaluate the risk and verify the triage of all reported adverse cases to the medical devices. The adverse events of medical devices could be device or equipment malfunction or unavailability, misused devices, user error, or any other cases that are suspect of being a major contributor to the adverse events. The process of obtaining a report to assess the triage requires three to five days, and the triage verification will allow MHRA to focus on the matters that present the greatest risk to the patient safety, and where their involvement will help to resolve the issue. Then MHRA will issue an alert or warning if necessary, after the investigation and risk evaluation has been completed. There are two kinds of alert or warning regarding medical devices: (1) field safety notifications- producer matters, recalling medical devices or equipment for technical or clinical reasons; (2) medical device alerts - providing information about medical device recalls and recommending what necessary steps and actions NHS hospitals should take [39]. The entire procedures make certain that each event report not only allows MHRA to learn about the medical device and how it uses but also takes proper measures to avoid the recurrence of similar problems. SPC charts can be applied to monitor the number of adverse events of medical devices and to examine whether the number of adverse events has successfully been reduced by MHRA and NHS.

\subsubsection{Methods}

On 27 June 2019, all recorded adverse events related to medical devices or equipment that took place in England from 1 January 2009 to 30 September 2018 were extracted using Excel 2016 from the NRLS database. NRLS summarized and compiled the submitted data from all NHS organizations, physicians, patients, nurses, and staff, then published the quarterly 
National Patient Safety Incident Reports. All reported adverse events with medical devices will be analyzed and examined using Minitab 18. As the sample size is not consistent, the U chart was chosen to keep track of the rate of adverse events per incident. Hence, the control limit lines will be specific for each data point.

\subsubsection{Data discussion}

Before the Department of Health released the report with the recommendation, the baseline period, from January 2009 to June 2012, there were a total of 130,512 adverse events with medical devices, with a total incident of 4,328,100 for this period. The mean rate of adverse events with medical devices per incident was around 0.030155 . The U control chart with the CL at $\bar{U}=0.030155$, the LCL and UCL are shown in Fig. 5. Three points fall outside the control limits, sample 2 (Apr 2009 - June 2009), 7 (Jul 2010 - Sep 2010), and 12 (Jan 2012 Mar 2012); hence, the process is not in the state of control. Such points need to be analyzed if a special cause can be identified.

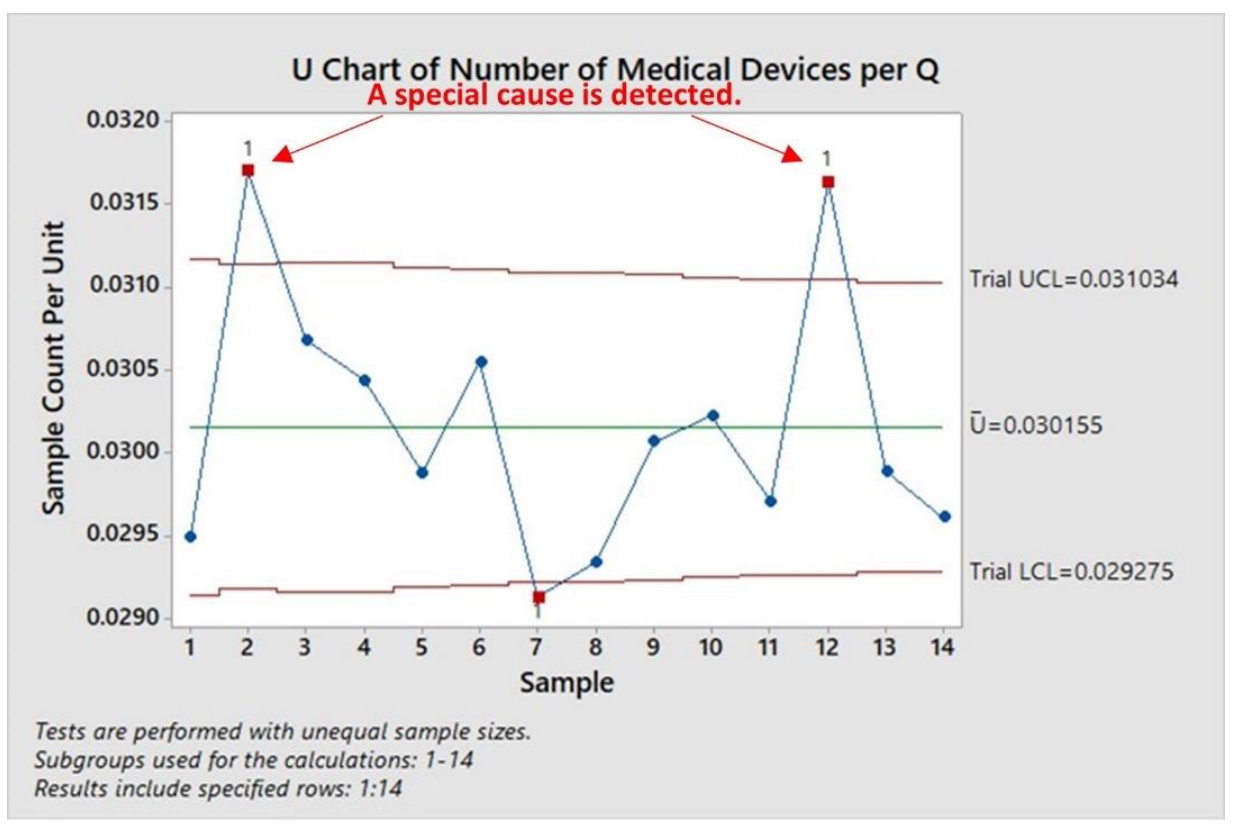

Fig. 5. $U$ chart for the average rate of adverse events in medical devices per incident before the recommendation from the Department of Health.

Investigation of sample 2 data, for the period from April 2009 to June 2009, discloses three medical device alerts were issued in April 2009, and one was issued in June 2009. Furthermore, the defective PIP breast implants, failure of stapler loading units in thoracic surgery resulted in the exceptionally high average rate of adverse events per incident in sample 12 data, for the period from October 2011 to December 2011. The following actions for these two sample points have been suggested by MHRA to prevent the recurrence of similar events. Moreover, no special causes can be determined for the out-of-control signal from sample 7 (July 2010 - September 2010); this sample will therefore be maintained. 
Sample 2 and 12 are then removed, and the revised centerline will be determined as $\mathrm{CL}=$ $\bar{U}=\frac{\sum_{j=1}^{1} C_{j}+\sum_{j=3}^{11} C_{j}+\sum_{j=13}^{14} C_{j}}{\sum_{j=1}^{1} m_{j}+\sum_{j=3}^{11} m_{j}+\sum_{j=13}^{14} m_{j}}=\frac{110,927}{3,709,650}=0.029902$.

Fig. 6 illustrates the revised CL and control limits, which $\mathrm{CL}=\bar{U}=0.029902$. Both the sample 2 and 12 are not removed from the control chart but omitted from control limits calculations. Also, notice the sample 7 are now fall within the revised control limits, the process is said to be in a state of control. Thus, the revised control limits in Fig. 6 can be applied to monitor the continuation of data after the recommendation for the Department of Health (Sample 15 - 23), from July 2012 to September 2014, to check if the first change in a process has enhanced the steady process (Phase II study).

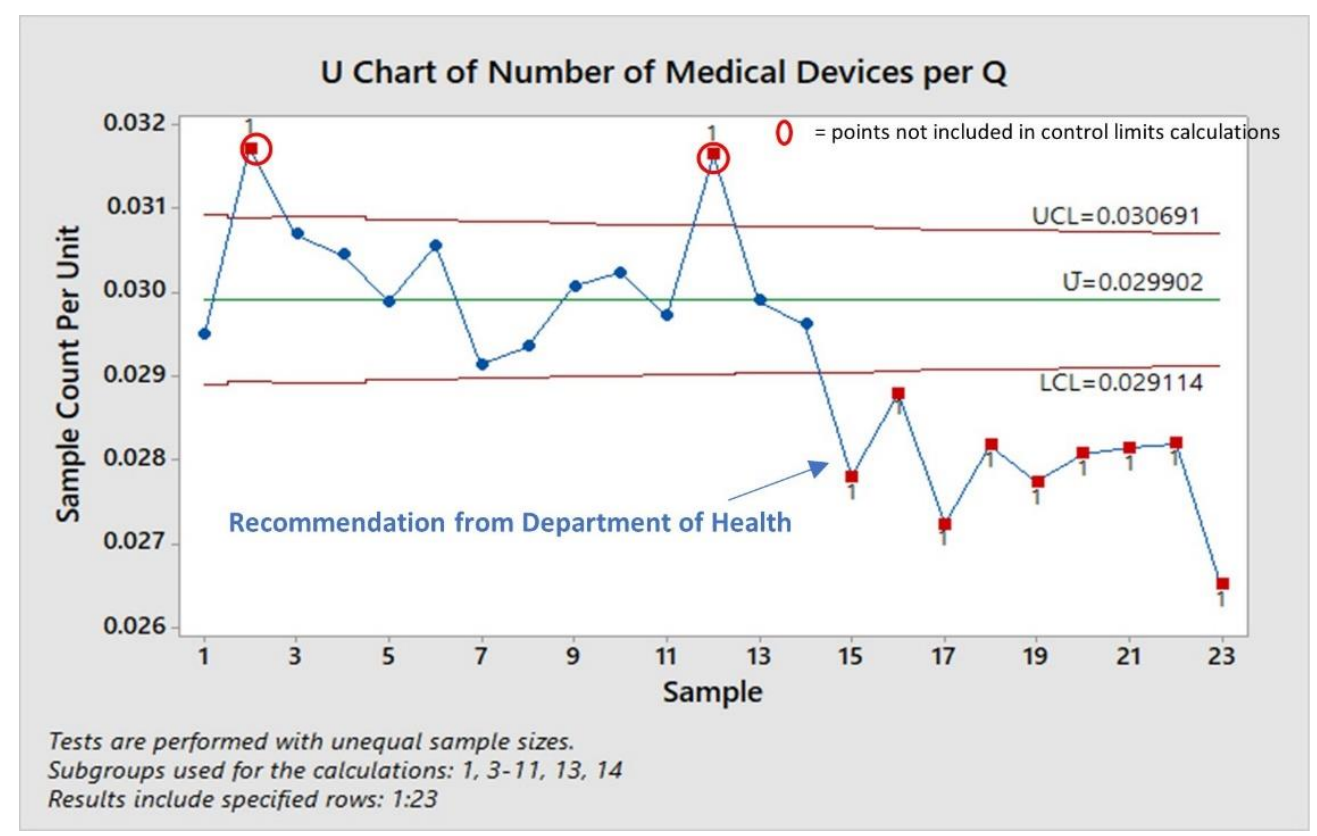

Fig. 6. Revised CL and control limits and continuation of U chart in Fig. 5.

Fig. 6 illustrates the data point for samples 15 to 23 fall below the revised lower control limit; thus, the process is out-of-control. It indicates that the process after the recommendation is now operating at a new level that is substantially better than the baseline level of $\mathrm{CL}=\bar{U}=0.029902$. Therefore, new control limits needed to be calculated again based on the data after the recommendation from July 2012 to September 2014 (sample 15 - 23). The new centerline will be determined at $\mathrm{CL}=\bar{U}=\frac{\sum_{j=15}^{23} C_{j}}{\sum_{j=15}^{23} m_{j}}=\frac{98,997}{3,557,908}=0.027824$, and the new CL and control limits are illustrated in Fig. 7. 


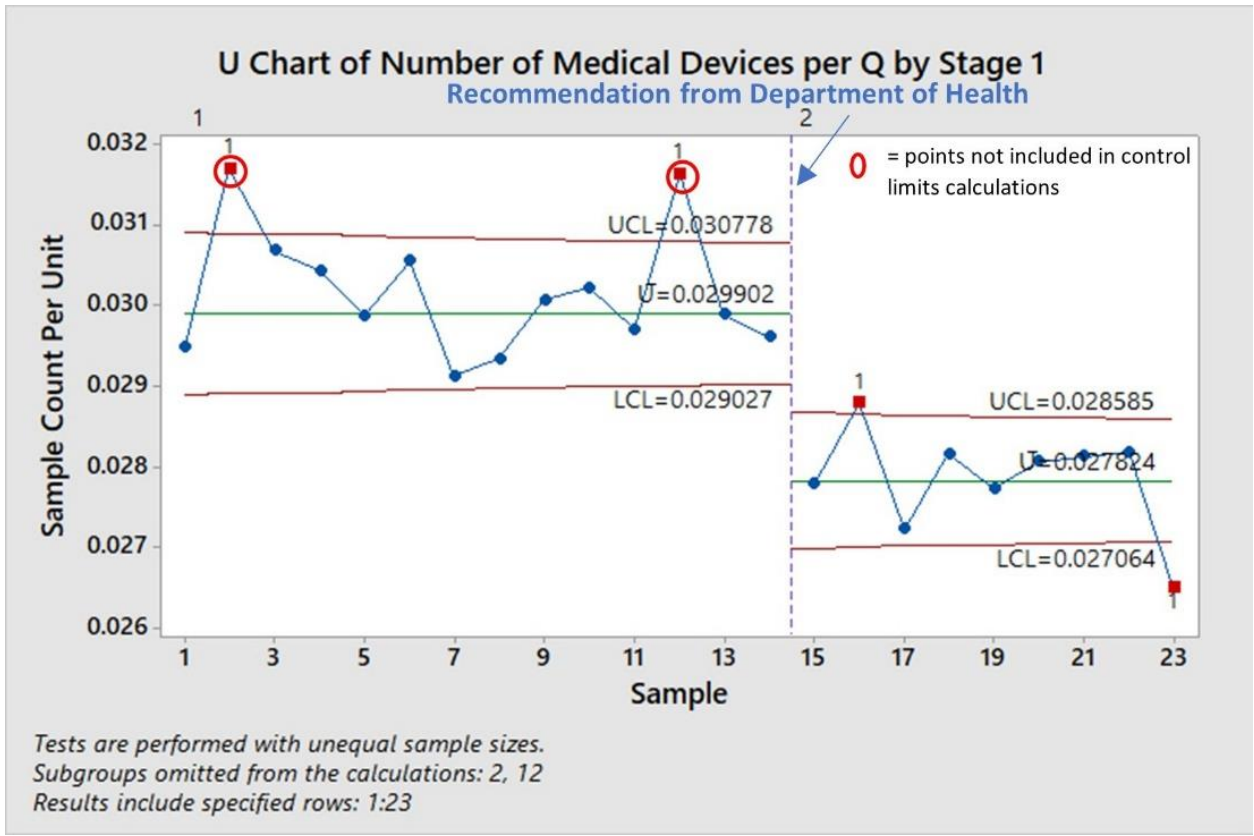

Fig. 7. New control limits on the average rate of adverse events in medical devices per incident.

Fig. 7 shows sample 16 (Oct 2012 - Dec 2012) fall above and 23 (Jul 2014 - Sep 2014) fall below the control limits, and thus, the process is out-of-control. Examination of the data from sample 16 reveals the uncommon high average rate of adverse events in medical devices is triggered by a malfunction of the external pacemaker with temporary pacing wires and emergency public access external defibrillator. And MHRA has issued an alert to suggest interventions to prevent the recurrence of similar events. For the out-of-control indication from sample 23, there are no special causes that can be detected; thus, this sample will be maintained. Sample 16 will therefore be excluded and the newly revised centerline will be determined as $C L=\bar{U}=\frac{\sum_{j=15}^{15} C_{j}+\sum_{j=17}^{23} C_{j}}{\sum_{j=15}^{15} m_{j}+\sum_{i=17}^{23} m_{j}}=\frac{88,370}{3,188,726}=0.027713$.

Fig. 8 illustrates the new revised CL and control limits, and the process now is in a state of control. Hence, the new revised control limits in Fig. 8 can be applied to monitor the continuation of data after the MHRA and NHS form partnership (sample 24 - 39), from October 2014 to September 2018. 


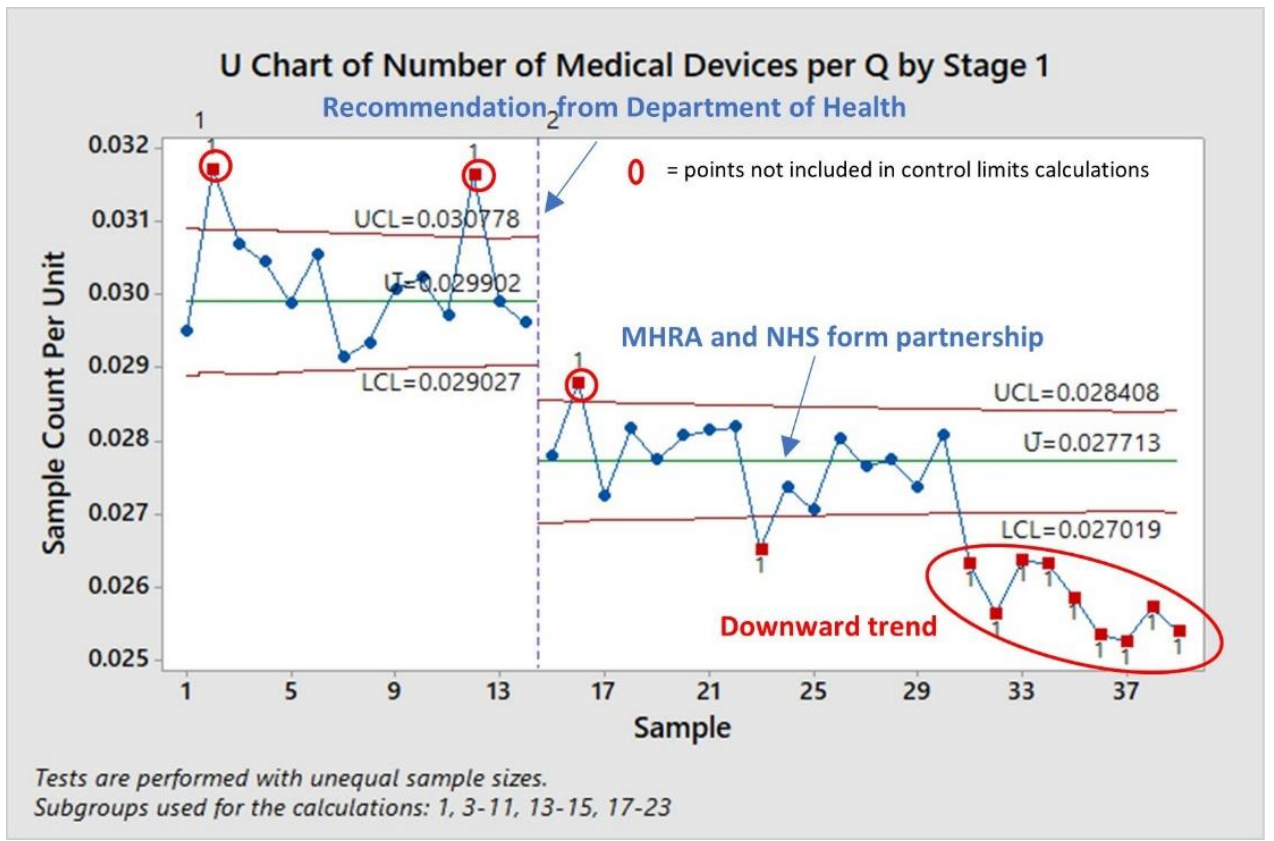

Fig. 8. New revised control limits on the average rate of adverse events in medical devices per incident.

Fig. 8 shows that the process is not in control and there has been a downward trend since July 2016 (sample 31), which possibly an indication that the process has changed from July 2016 to September 2018 (sample 31 - 39). The control chart indicates an improvement; perhaps not because of the partnership between MHR and NHS which formed in September 2014, but because of some other factor which started in July 2016. Thus, the new control limits needed to be calculated again based on the data from July 2016 to September 2018 (sample $31-39$ ). The new centerline will be determined at $\mathrm{CL}=\bar{U}=\frac{\sum_{j=31}^{39} C_{j}}{\sum_{j=31}^{39} m_{j}}=\frac{119,646}{4,640,404}=$ 0.025784, the complete control chart is shown in Fig. 9.

Fig. 9 indicates that the has process has improved immediately to a new level from $\bar{U}=$ 0.029902 to 0.027713 after the Department of Health recommendation in June 2012 (sample 14). It also indicates that the average rate of adverse events in medical devices per incident decreased from $\bar{U}=0.027713$ to 0.025784 between July 2016 to September 2018 (sample 31 - 39), and the process is consistent during this period. The decrease, however, was not due to the partnership between MHRA and NHS that was established after September 2014 (sample 24), but due to other factors that started in July 2016. Hence, the NHS and MHRA partnership does not seem to have any influence on decreasing the average rate of adverse events in medical devices. Although the partnership has not resulted in improvement, the control chart can assist MHRA and NHS reduce unnecessarily or avoid wasted investments and resources in introducing ineffective change. During the period from July 2016 to September 2018 (sample 31 - 39), the control chart indicates that the process is in a control state; thus, the process during this period displays only common cause variation. If MHRA and NHS seek to reduce the average rate of adverse events in medical devices per incident and minimize the variation, further investigation and implementation of other improvement strategies are necessary. Consequently, the next step for the NHS and MHRA is to assess an improvement proposal, continue the application of the control chart to evaluate the new 
process with the existing measurements, and verify if the process has improved, unchanged, or getting worse.

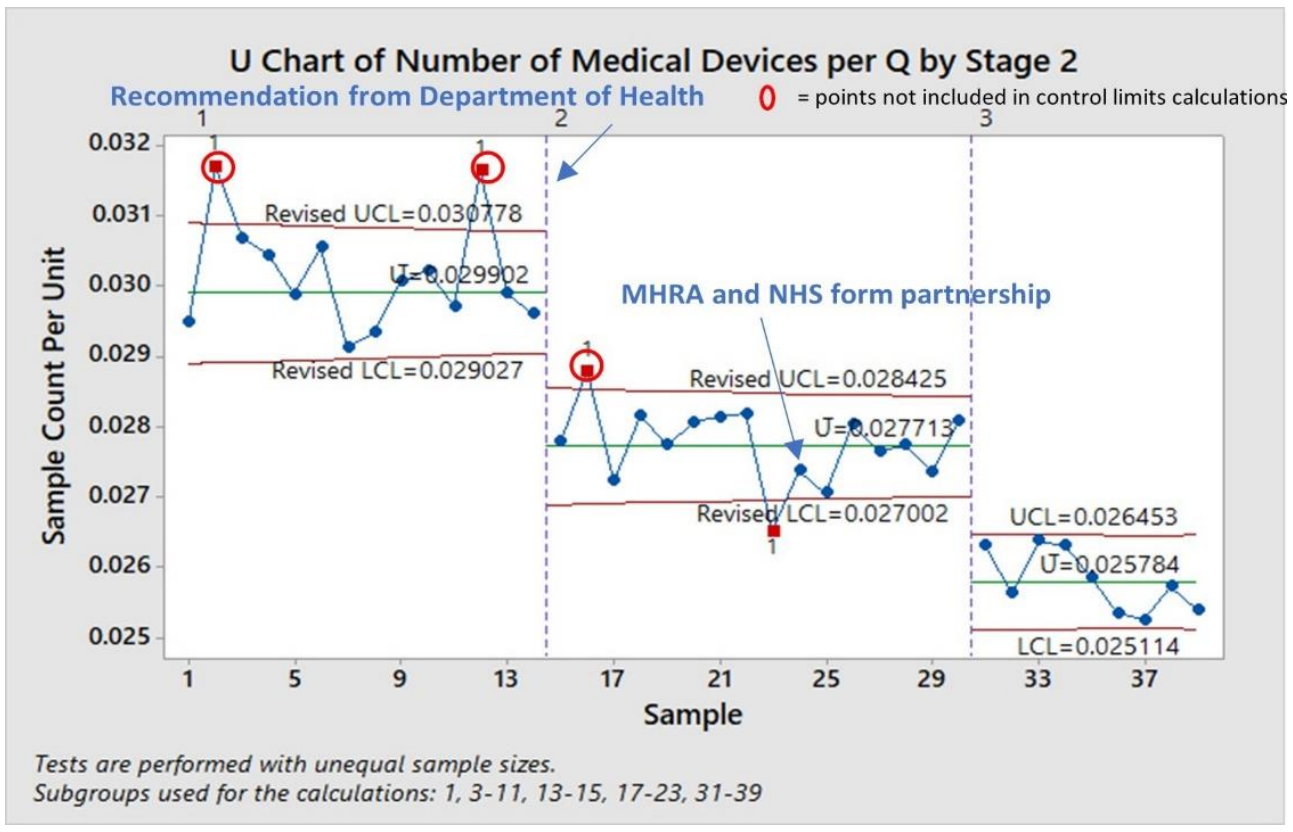

Fig. 9. Complete $U$ chart on the average rate of medical device adverse events per incident.

\subsection{Control Chart methodology discussion}

The examples above show common ideas of control charts. The key benefit of using the methodology of control charts is it can identify whether the process is in a state of control and to determine whether there is a special cause variation or significant signal. The control charts can help healthcare institutions to avoid wasted investments and resources in any changes that sound fantastic but have no positive impact on actual improvement, as in the case of the medical devices/equipment incidents example. Also, the control charts can detect a significant indication or process change from the data pattern more rapidly than other statistical tools. Generally, the traditional method of statistical analysis is based on statistical hypothesis testing of a large number of samples from a population disregarding chronological or time order- for instance, the ambulance response time before (pre) change might be compared with after (post) change. Hypothesis testing or significant tests are the statistical approaches that are frequently applied to assess if the differences between the two groups are statistically significant. This approach is only effective if there is a sufficiently large amount of data; nevertheless, not all of us have the resources and time to collect and gather largescale data. Using these traditional approaches in healthcare research is always limited by the delay in collecting sufficient data on a large scale and researchers might switch to implement other simple approaches such as bar graphs, line charts, tables, or pie charts for presenting the data. Researchers can only make a qualitative observation in such a situation in identifying whether the change brings real improvement. The control charts implementation, by contrast, does not involve as much data as the traditional approach and the charts indicate how the process shifts over time with data plotted in chronological order. As a result, SPC charts can identify process patterns and trends faster. Generally, these examples illustrate 
how a control chart can help the healthcare organizations to choose the right and proper improvement plan-whether to detect and remove special causes of variation to bring the process back into the state of control (when the process is out of control) or to make more effort to enhance the fundamental process and to redesign the process in the desired direction (when the process is in control). Besides, the control chart, as indicated in the examples above, can also be used as a simple tool to keep track of whether the improved process is maintained over time. Thus, a control chart helps visualize the efficacy of the current process performance, taking more statistical rigor to the act of making critical decisions and ensuring the consistency of process improvements over time.

\section{Limitation}

The purpose of this study is to perform a study of the application of control charts in monitoring healthcare performance. This research involves studying various types and construction of control charts in tracking healthcare performance and then being able to determine the most suitable control chart on the relevance of performance in healthcare. Healthcare performance evaluation is based solely on the secondary data within the healthcare organizations. The evaluation of healthcare performance is completely based on the current secondary data within the healthcare institutions. This study is intended to assist the healthcare institutions to decrease variability and inconsistency and save significant time and associated operational costs by applying the performance monitoring control chart.

Due to the time limit and cost-effectiveness, secondary data analysis was used in this research. Furthermore, it is often recommended to have 20-25 data points to determine the control limits [19]. Nevertheless, we did not participate in the data collection process; hence, we do not influence how and what the data is collected. Therefore, we are unable to increase the number of observations. Moreover, this research considered only the two types of the methodology of control charts that will have been applied frequently in the healthcare sector.

\section{Conclusion}

This study will indicate the importance of control charts as an effective monitoring tool in monitoring and enhancing the process performance over time by analyzing statistical variation and its source. When there is greater human participation in healthcare, the chances of error are also greater. Control chart aids to detect the source of error by differentiating the common and special cause variation, each requiring a different response from healthcare management. The healthcare organization should then be aware of any unusual behavior or out of statistical control condition that occurs in the process and preventive action might be taken immediately to eliminate the source of variation. It helps to ensure that the process is in control and constantly working at its fullest potential by monitoring a process. The constructed control chart managed to bring a lot of benefits to the healthcare industry as it offers convenience and flexibility to healthcare organizations to prevent organizations from spending additional time and resources in guiding employees and from adopting an unproductive change which can contribute to unsuitable decision making throughout the hospital. The control chart is one of the quality improvements approaches that can be used to achieve sustainable improvement by the staff or physicians. A high-quality healthcare system is essential for a nation as it indicates that the population has the ability to combat disease; it provides a country with the necessary stability to develop an effective and reliable workforce 
that boosts productivity and leads to a more unified nation. This contributes significantly to economic development and helps to create a robust economy.

The author would like to express gratitude to NHS England for conducting the healthcare data and sharing this data with the public.

\section{References}

1. World Health Organization, Ageing and health (2018). Available at:

< https://www.who.int/news-room/fact-sheets/detail/ageing-and-health> [Accessed 30 October 2018]

2. J. Arthur, Lean six sigma for hospitals, Improving patient safety, patient flow and the bottom line, 2nd ed. (McGraw-Hill Education, New York, 2016)

3. G. Suman, D. Prajapati, Int. J. Metrol. Qual. Eng. 9, 5 (2018)

4. P.E. Oguntunde, O.A. Odetunmibi, O.O. Oluwadare, Int. J. Sci. Res. 14, $154-158$ (2015)

5. T. Love, N. Ehrenberg, Sapere Research Group, Addressing unwarranted variation: literature review on methods for influencing practice [Report], HQSC, 25 March 2014, Wellington, New Zealand (2014)

6. W.K. Chang, H.K. Paul, Basic statistical tools for improving quality (John Wiley \& Sons Ltd, New Jersey, 2012)

7. F. Faltin, R. Kenett, R. Ruggeri, eds, Statistical methods in healthcare (John Wiley \& Sons Ltd, New Jersey, 2012)

8. M. Owen, SPC and continuous improvement (Springer Science \& Business Media, Berlin, 2013)

9. J.M. Levett, R.G. Carey, Ann. Thorac. Surg. 68, 353 - 358 (1999)

10. J.C. Benneyan, R.C. Lloyd, P.E. Plsek, BMJ Qual. Saf. 12, 458 - 464 (2003)

11. D.L. Bush, JHQ, 13, $40-48$ (1991)

12. E. Curran, P. Harper, H. Loveday, H. Gilmour, S. Jones, J.H. Benneyan, R. Pratt, J. Hosp. Infect. 70, $127-135$ (2008)

13. L.J. Finison, K.S. Finison, C.M. Bliersbacl., J. Healthc. Qual. 15, 9 - 22 (1993)

14. L.J. Finison, K.S. Finison, J. Healthc. Qual. 18, 32 - 41 (1996)

15. C.P. Quesenberry, Am. J. Infec. Control 28, $314-320$ (2000)

16. L. VanderVeen, JHQ, 14, 20 - 29 (1992)

17. M.A. Mohammed, P. Worthington, W.H. Woodall, Qual. Saf. Health Care 17, 137 145 (2008)

18. P. Wisner, Statistical process control for quality improvement (University of Tennessee, USA, 2005)

19. D.C. Montgomery, Introduction to statistical quality control (John Wiley \& Sons Ltd, New Jersey, 2009)

20. W.A. Shewhart, Economic control of quality of manufactured product (D. Van Nostrand Company, New York, 1931)

21. S.G. Amin, Qual. Manag. Health Ca. 9, 1 - 27 (2001)

22. J.C. Benneyan, Infect. Cont. Hosp. Ep. 19,194 - 214 (1998) 
23. J.C. Benneyan, Int. J. Six Sigma Competitive Advant. 4, 209 - 239 (2008)

24. J. Murdoch, J.A. Barnes, Statistical Tables, $4^{\text {th }}$ ed. (UK: Palgrave Macmillan, 1998)

25. NHS England, Ambulance quality indicators (2019). Available at: $<$ https://www.england.nhs.uk/statistics/statistical-work-areas/ambulance-qualityindicators/ > [Accessed 18 June 2019]

26. NHS England, New ambulance standards - easy read document (2017). Available at: $<$ https://www.england.nhs.uk/publication/new-ambulance-standards-easy-readdocument/ > [Accessed 18 June 2019]

27. NHS England, New ambulance service standards announced (2017). Available at: $<$ https://www.england.nhs.uk/2017/07/new-ambulance-service-standards-announced/ $>$ [Accessed 22 June 2019]

28. National Health Executive, NHS England to change 'inefficient' ambulance response targets, 21 March 2017, UK (2017)

29. North East Ambulance Service, Understanding ambulance response categories (2017). Available at: < https://www.neas.nhs.uk/our-services/accident-emergency/ambulanceresponse-categories.aspx > [Accessed 22 June 2019]

30. NHS England, Ambulance response program (2019). Available at: $<$ https://www.england.nhs.uk/urgent-emergency-care/improving-ambulanceservices/arp/> [Accessed 22 June 2019]

31. Nuffield Trust, Ambulance response times (2019). Available at: < https://www.nuffieldtrust.org.uk/resource/ambulance-response-times> [Accessed 19 June 2019]

32. J. Ward, P.J. Clarkson, J. Med. Eng. Technol. 28, 2 - 21 (2004)

33. P. Davenport, Adverse incident reporting and the MHRA, PMG, UK (2019)

34. Axenics, Meeting the increased demands of the medical device manufacturing industry (2017). Available at: <https://axenics.com/blog/meeting-increased-demands-medicaldevice-manufacturing-industry> [Accessed 30 October 2018]

35. B. Russell, PIP breast implant scandal: Victims visited by bailiffs, BBC News, 28 May 2019, England (2019). Available at: <https://www.bbc.com/news/uk-england48132572> [Accessed 10 July 2018]

36. NHS Choices, PIP breast implants - latest from the NHS (2012). Available at: < https://www.nhs.uk/news/2012/01January/Pages/government-review-advises-onfrench-pip-breast-implants.aspx > [Accessed 10 July 2019]

37. NHS England, Patient safety alert, stage three: directive, improving medical device incident reporting and learning, 20 March 2014, UK (2014)

38. T. Stephenson, Expert clinical advice - MHRA Medical Devices (2014). Available at:<https://www.pmguk.co.uk/data/page_files/publications\%20and\%20reports/2014/co n402542.pdf $>$ [Accessed 11 July 2018]

39. M. Thompson, C. Heneghan, M. Billingsley, D. Cohen, BMJ, 342, 1131 - 1132 (2011) 